University of Wollongong

Research Online

Faculty of Informatics - Papers (Archive)

Faculty of Engineering and Information

Sciences

2009

\title{
A fringe period unwrapping technique for digital fringe profilometry based on spatial shift estimation
}

Pu Cao

University of Wollongong

Jiangtao Xi

Faculty of Informatics, University of Wollongong, jiangtao@uow.edu.au

Joe F. Chicharo

University of Wollongong, chicharo@uow.edu.au

Yanguang Yu

University of Wollongong, yanguang@uow.edu.au

Follow this and additional works at: https://ro.uow.edu.au/infopapers

Part of the Physical Sciences and Mathematics Commons

\section{Recommended Citation}

Cao, Pu; Xi, Jiangtao; Chicharo, Joe F.; and Yu, Yanguang: A fringe period unwrapping technique for digital fringe profilometry based on spatial shift estimation 2009.

https://ro.uow.edu.au/infopapers/3297

Research Online is the open access institutional repository for the University of Wollongong. For further information contact the UOW Library: research-pubs@uow.edu.au 


\title{
A fringe period unwrapping technique for digital fringe profilometry based on spatial shift estimation
}

\author{
Abstract \\ In this paper we present a review of the phase unwrapping problem in Fringe Pattern Profilometry (FPP), \\ based on which we study the spatial shift wrapping problem in spatial shift estimation (SEE) based FPP. \\ An approach for carrying out the spatial shift unwrapping is proposed with its performance confirmed by \\ experiments. \\ Disciplines \\ Physical Sciences and Mathematics

\section{Publication Details} \\ Cao, P., Xi, J., Chicharo, J. F. \& Yu, Y. (2009). A fringe period unwrapping technique for digital fringe \\ profilometry based on spatial shift estimation. In P. Huang, T. Toshizawa \& K. Harding (Eds.), Optical \\ Inspection and Metrology for Non-Optics Industries, Proceeding of SPIE: Vol.7432 (pp. \\ 743208-1-743208-11). Washington, USA: SPIE.
}




\title{
A Fringe Period Unwrapping Technique for Digital Fringe Profilometry based on Spatial Shift Estimation
}

\author{
$\mathrm{Pu}$ Cao, Jiangtao $\mathrm{Xi}^{*}$, Joe F. Chicharo and Yanguang Yu \\ School of Electrical, Computer and Telecommunications Engineering \\ University of Wollongong, Wollongong, NSW2522, Australia
}

\begin{abstract}
In this paper we present a review of the phase unwrapping problem in Fringe Pattern Profilometry (FPP), based on which we study the spatial shift wrapping problem in spatial shift estimation (SEE) based FPP. An approach for carrying out the spatial shift unwrapping is proposed with its performance confirmed by experiments.
\end{abstract}

Keywords: fringe pattern profilometry, 3D measurement, phase unwrapping

\section{Introduction:}

In recent years, fringe pattern profilometry (FPP) has attracted increasing research effort as an enabling technology for non-contact measurement of three-dimensional (3D) object surfaces. Among various system implementation schemes for FPP, the one based on digital video projection (DVP) is particularly attractive due to the advantages of simple system structure and controllable fringe patterns.

Figure 1 shows the system structure of a DVP based FPP, consisting of a digital video projector, a CCD camera and a reference plane. With the system, a frame of image with a particular fringe pattern is produced by the digital projector and projected onto the reference plane, and then onto the surface of the object when the reference plane is removed. The projected images from the reference plane and the object surface are captured by the CCD camera, with the later being a deformed version of the former by the variance of the height of the object surface. As the deformed fringe pattern carries the information of surface shape, 3D profile of the object can be retrieved from these two fringe patterns.

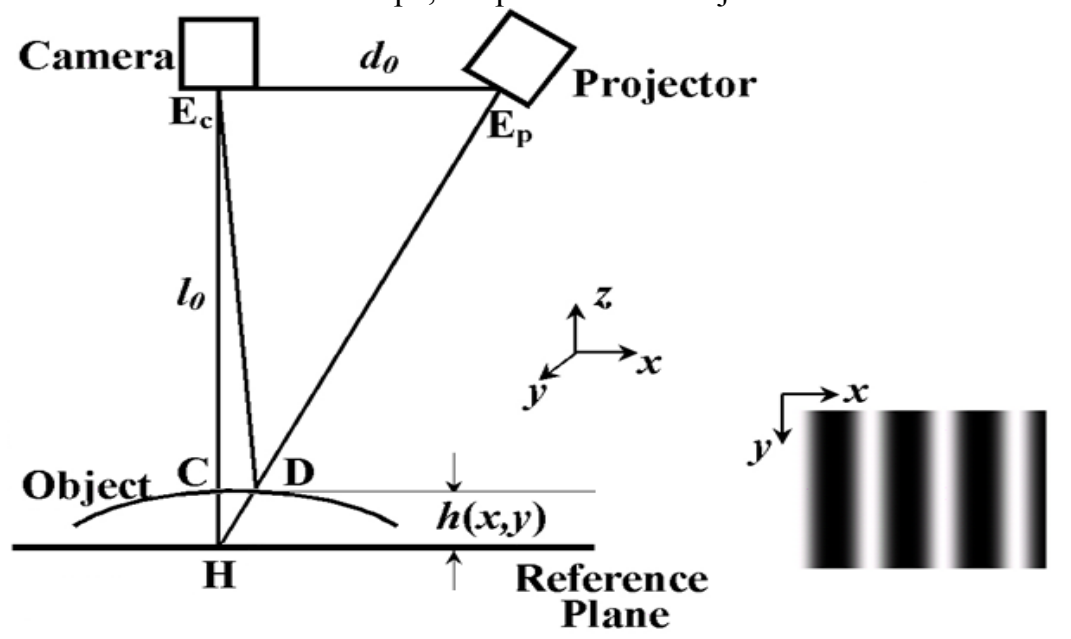

Figure 1. Schematic diagram of FPP system.

*jiangtao@uow.edu.au; phone 612 42213412; fax 61242213236

Optical Inspection and Metrology for Non-Optics Industries, edited by Peisen S. Huang,

Toru Yoshizawa, Kevin G. Harding, Proc. of SPIE Vol. 7432, 743208 • (c) 2009

SPIE · CCC code: 0277-786X/09/\$18 - doi: 10.1117/12.824845 
Several FPP approaches have been developed during the past decades. The most widely used are these based on phase difference estimation (PDE). In these approaches, the projected fringe patterns are sinusoidal or periodic, and the deformed one reflected from the object surface is considered as the result of phase modulation of the original fringe pattern. The surface profile is obtained by detecting the phase maps of the two fringe patterns. A number of fringe pattern analysis methods have been developed, such as Fourier transform profilometry (FTP) [1], phase shifting profilometry (PSP), phase measuring profilometry (PMP) [2, 3, 4], modulation measurement profilometry (MMP) [5], spatial phase detection (SPD) [6,7], phase lock loop (PLL) profilometry [8], Moire technique (MT) [9], laser triangulation measurement [10], colour-coded fringe projection [11,12] and other methods [13, 14]. Among existing approaches, FTP and PSP are most popular and widely used.

Although phase based approaches have been considered as the most popular, they suffers from a number of weaknesses. A major restriction is that fringe patterns must be either sinusoidal or ideal periodic. However such a requirement is hard to meet in practice due to some factors, such as the nonlinear distortion on inherent to digital video projections. In order to solve the problem, a profilometry approach was proposed by $\mathrm{Hu}$, et al $[15,16]$, which, instead of detecting the differences between the phase maps, is based on the estimation of spatial shift for corresponding pixels on the two fringe patterns. The approach is referred to as spatial shift estimation (SSE) profilometry approach, or Generalized Analysis Model (GAM) based approach [15][16].

Phase unwrapping is a major problem associated with FDE-based FPP approaches. This problem arises because the phase difference can only be detected within the main value range of $[-\pi, \pi]$, but the true phase difference can be arbitrary. In order to retrieve the actual surface shape of the object, phase unwrapping must be carried out to obtain the actual phase maps. Many methods have been proposed to solve the unwrapping problem in FDE approach [17][18]. In the SSE-based approaches, spatial shift between corresponding pixels on the two fringe patterns can also be arbitrary, it can only be detected without ambiguity within the range of $[0, T]$, where $T$ is the width of the individual fringe. Obviously, shift unwrapping is also required in order to correctly restore the 3D shape of the object surface. However, spatial shift unwrapping for SSE-based FPP is still an outstanding issue, which motivated the work presented in this paper.

This paper is organized as follows. In Section 2 we firstly present a brief description of conventional PDE and SSE based FPP, including working principles, system structures and relevant algorithms. Then in Section 3 we review the phase unwrapping problem in PDE based approaches, based on which we indicate that a similar problem exists in SSE approach. Section 4 presents a method for solving the problem. Finally in Section 5 experimental results are presented to demonstrate the effectiveness of the proposed method.

\section{Principle of Fringe Pattern Profilometry}

\subsection{Principle of Triangulation}

FPP is based on the triangulation principle described as follows. As the image produced by the projector has a fringe structure, without loss of generality we can assume that light intensity varies periodically alone $x$ direction, while keeping constant along $y$ direction, as shown in Figure 1. We can use $s(x), d(x)$ and $h(x)$ to denote the variance of light intensity of the fringe pattern on the reference plane and object surface as well as the height distribution along $x$ coordinate respectively. We also assume that the reference plane and the object surface have the same reflective characteristics.

Let us consider what happens when a beam of light is projected onto the point $\mathrm{D}$ on the object. When the object is removed, the same light beam (hence with the same intensity) should be projected onto point $\mathrm{H}$ on the reference surface, which is reflected back to the camera through point C. As the triangles $E_{c} E_{P} H$ and $C D H$ are similar, we have the following relationship:

$$
\frac{d_{0}}{l_{0}}=\frac{\overline{C D}}{h\left(x_{d}\right)}
$$

Note that $x_{d}$ denotes the coordination positions of point D. $h\left(x_{d}\right)$ denotes the distance between points $\mathrm{C}$ and the reference plane, given by: 


$$
h\left(x_{d}\right)=\frac{l_{0} \overline{C D}}{d_{0}}
$$

The above relationship gives the foundation for FPP.

\subsection{PDE based approaches for FPP}

The FDE based FPP utilize fringe patterns that are periodic and can be expressed as [19, 20]:

$$
s(x)=\sum_{k=0}^{+\infty} b_{k} \cos \left(2 \pi k f_{0} x+\psi_{k}\right)
$$

and the deformed fringe pattern can also be expressed as:

$$
d(x)=\sum_{k=0}^{+\infty} b_{k} \cos \left(2 \pi k f_{0} x+\phi_{k}(x)+\psi_{k}\right)
$$

In the above equations, $f_{0}$ is the spatial frequency of the fundamental component in the fringe patterns, and $b_{k}$ is the amplitude of the $k$-th order harmonic component. $\psi_{k}$ is the initial phase of the $k$-th order harmonic component, and $\phi_{k}(x)$ denotes the phase difference between the $k$-th order harmonic components of these two fringe patterns.

Equations (3) and (4) show that $s(x)$ and $d(x)$ are related by the phase shift $\phi_{k}(x)$. Let us consider the light beam projected at point $\mathrm{D}$ on the object and $\mathrm{H}$ on the reference plane when the object is removed. The phase shift between $\mathrm{C}$ and $\mathrm{D}$ can be determined by the spatial distance $\overline{C D}$, and hence we have $[17,18]$ :

$$
\phi_{k}\left(x_{d}\right)=2 \pi k f_{0} \overline{C D}=k \cdot 2 \pi f_{0} \overline{C D}=k \cdot \phi\left(x_{d}\right)
$$

where $\phi\left(x_{d}\right)=2 \pi f_{0} \overline{C D}$ is the phase shift of the fundamental component.

Substituting Equation (5) to Equation (2) we have:

$$
h\left(x_{d}\right)=\frac{l_{0} \phi\left(x_{d}\right)}{2 \pi f_{0} d_{0}}
$$

As points $\mathrm{D}$ and $\mathrm{H}$ are arbitrary, the derivations should apply to all the points on the projected fringe pattern. Therefore we have:

$$
h(x)=\frac{l_{0} \phi(x)}{2 \pi f_{0} d_{0}}
$$

Equation (7) shows that as long as the gives $\phi(x)$ can be detected, we are able to calculate the height distribution $h(x)$ of the object surface. This is the foundation of all PED based approaches.

\subsection{Spatial Shift Estimation based FPP}

The FDE based FFP methods suffer from some limitations. In particular, the fringe pattern used to project is limited to be sinusoidal or purely periodic in order that the phase maps of $s(x)$ and $d(x)$ exist and can be detected. However, due to many undesired factors inherent to digital projection, such as geometrical distortion and nonlinear intensity distortion, purely sinusoidal fringe patterns are hard to produce. In order to solve these problems, Hu et al [15, 16] introduced a method which is based on the spatial shift estimation (SSE) rather than PSE. 
The SSE based approach is rather simple and straight forward. Let us consider $\overline{C D}$, the distance between $\mathrm{C}$ and $\mathrm{D}$ again, which is obviously a function of the location of $\mathrm{D}$ (i.e. $x_{d}$ ), the location of $\mathrm{H}$ (or C, i.e., $x_{c}$ ) and the height of the object at point $\mathrm{H} h\left(x_{d}\right)$. Therefore we have the following:

$$
\frac{d_{0}}{l_{0}}=\frac{u\left(x_{d}\right)}{h\left(x_{d}\right)}
$$

where $u\left(x_{d}\right)=\overline{C D}=x_{c}-x_{d}$, which is the spatial distance between $x_{d}$ and $x_{c}$. Note that $x_{d}$ and $x_{c}$ are the points on $d(x)$ and $s(x)$ having the same light intensity, that is $d\left(x_{d}\right)=s\left(x_{c}\right)$. As the above derivation is valid for any $x_{d}$ and $x_{c}$, we can replace $x_{d}$ by $x$, yielding the following:

$$
h(x)=\frac{l_{0} u(x)}{d_{0}}
$$

Note that $u(x)$ is the spatial distance between a point $x$ on $d(x)$ and the corresponding point on $s(x)$ with the same light intensity, that is:

$$
d(x)=s(x-u(x))
$$

Equations (9) and (10) provide a straight forward way to obtain the 3D profile of the object surface. With $d(x)$ and $s(x)$ available, if we are able to obtain $u(x)$ to meet Equation (10), we then can utilize Equation (9) to yield $h(x)$, the height distribution of the object surface along $x$. By repeating the procedure for all $y$ we should be able to obtain the $3 \mathrm{D}$ profile of the object surface.

The spatial shift based approach has a particular advantage. The projected fringe patterns are no longer required to be sinusoidal, which implies that even there are distortions with the fringe patterns, sufficient three-dimensional information on the object surface is contained in the variation between projected and deformed fringe patterns. Thus the profilometry can be archived. This is the advantage of Generalized Model.

\section{The Unwrapping Problem}

In this part, we first review the unwrapping problem in conventional FDE and SSE.

\subsection{Phase Unwrapping in conventional FDE:}

From Equation (7) we have

$$
\phi(x)=2 \pi \frac{d_{0} h(x)}{T_{0} l_{0}}
$$

where $T_{0}=1 / f_{0}$ is the width of an individual fringe. Obviously, $\phi(x)$ can take any value, depending on $h(x)$, $d_{0}, l_{0}$ and $T_{0}$. However, with most PDE based approaches, $\phi(x)$ can only be identified within the range of $[-\pi, \pi]$. In other words, the phase is wrapped into the main value range. In the following such a wrapped phase is denoted as $\phi_{w}(x)$. Figure 2 shows an example which demonstrates the difference between $\phi(x)$ and $\phi_{w}(x)$. Assuming we have an object with its height distribution given in Figure 2 (a), the phase map $\phi(x)$ should be the one shown in Figure 2 (b) based on Equation (11). However, most PDE based approaches are only able to yield a $\phi_{w}(x)$ shown in Figure 2 (c). If such a wrapped $\phi_{w}(x)$ is used in Equation (7), we will obtain a height distribution in Figure 2 (d), which obviously suffers from significant errors. Consequently, in order to correct $h(x)$, we must employ $\phi(x)$ instead of $\phi_{w}(x)$. 

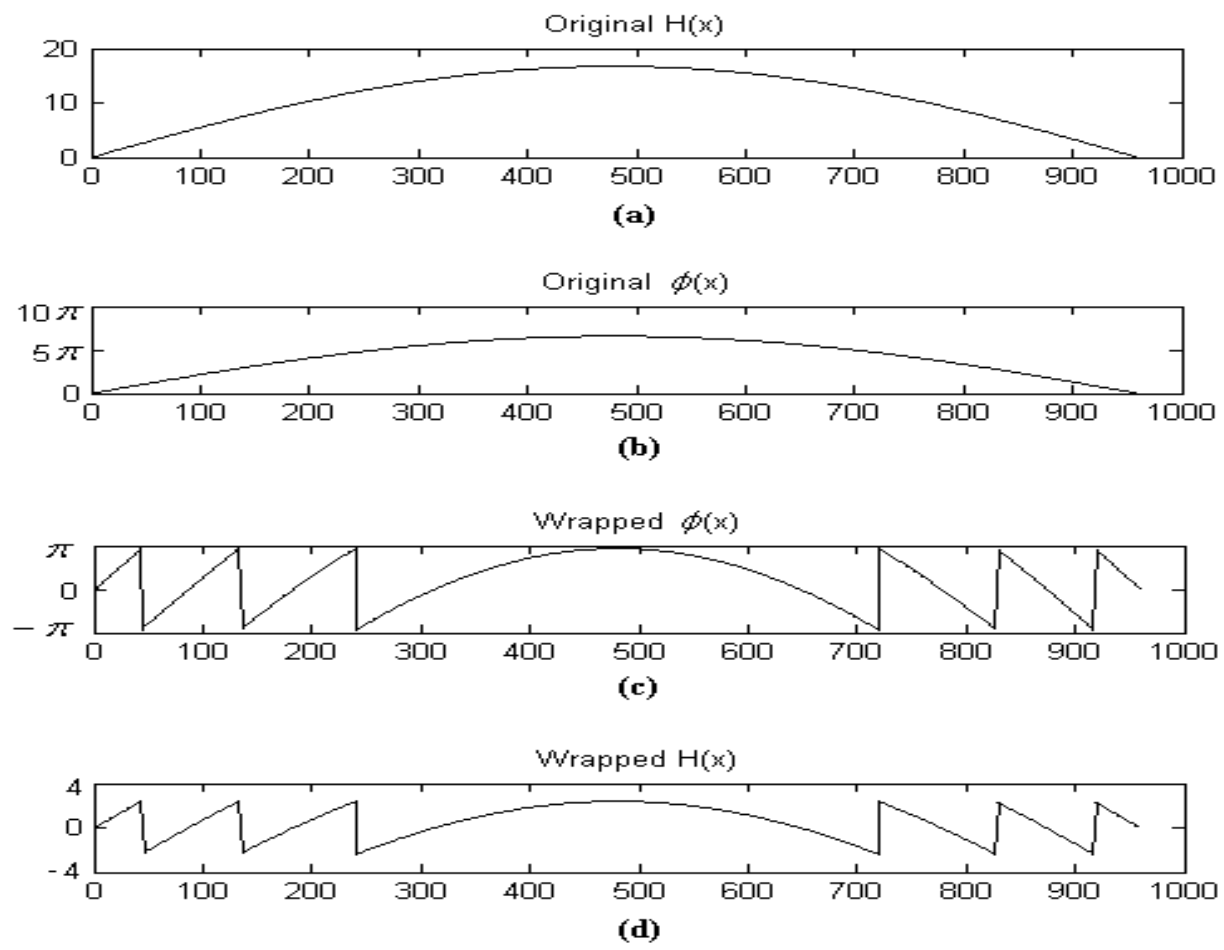

Figure 2. Unwrapped and wrapped phase maps

Phase unwrapping refers to the process of restoring $\phi(x)$ from $\phi_{w}(x)$. As can be seen by Figure 2, the phase is wrapped in the following way. When $\phi(x)$ increases and reaches points $\pi, 3 \pi, 5 \pi$,...(i.e., odd number multiples of $\pi), \quad \phi_{w}(x)$ drops from $\pi$ to $-\pi$. Similarly, when $\phi(x)$ varies decreasingly and reaches the same points, $\phi_{w}(x)$ jumps from $-\pi$ to $\pi$. The phase unwrapping should reverse the process. In other words, when we observe a phase drop from $\pi$ to $-\pi$, we should add $2 \pi$ to the unwrapped $\phi(x)$, and when we notice a phase jump from $-\pi$ to $\pi$, we should add $-2 \pi$.

\subsection{Spatial Shift Unwrapping in SSE based FPP:}

A wrapping problem also exists in SEE approaches. From Equation (9) we have:

$$
u(x)=\frac{d_{0} h(x)}{l_{0}}
$$

Depending on $h(x), d_{0}$ and $l_{0}$, the shift function $u(x)$ may take any value as well. However, when $s(x)$ has a fringe structure with a periodic fringe of width $T_{0}, u(x)$ can only be detected within the main value of $\left[0, T_{0}\right]$. In other words, $u(x)$ is wrapped into $\left[0, T_{0}\right]$, which is denoted as $u_{w}(x)$ and given as follows:

$$
u_{w}(x)=u(x)-k T_{0} \text {, where } k=\text { Integer }\left[\frac{u(x)}{T_{0}}\right]
$$

In order to demonstrate the relationship, we utilize the same example in Figure 2. With $h(x)$ shown in Figure 3 (a), we should have $u(x)$ in Figure 3 (b). However, what we have is $u_{w}(x)$ as shown by Figure 3 (c). Use of $u_{w}(x)$ in Equation (9) will result in significant error in $h(x)$, as shown by Figure $3(\mathrm{~d})$. Therefore, we must work out a way to restore $u(x)$. The process is referred to as spatial shift unwrapping. 


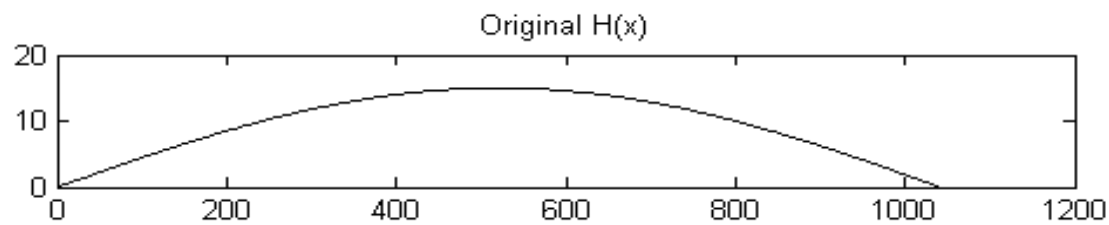

(a)

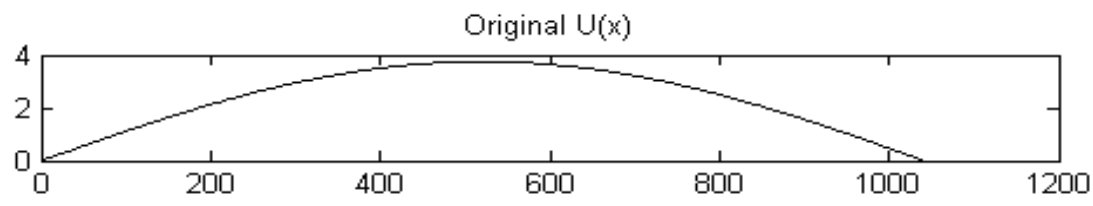

(b)

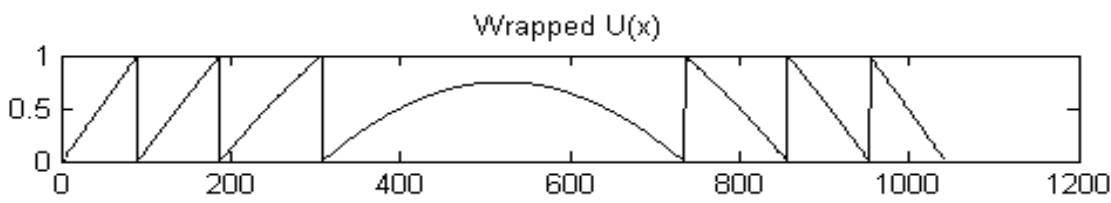

(c)

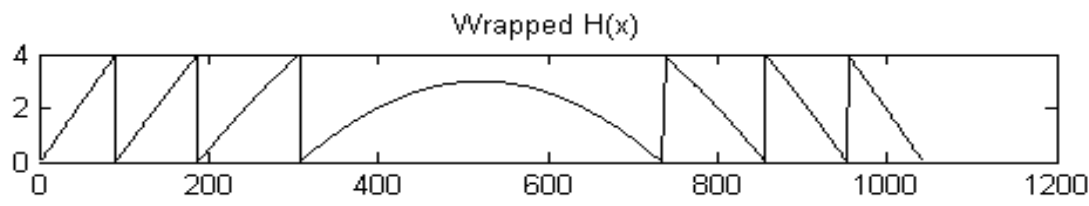

(d)

Figure 3. Unwrapped and wrapped shift maps

\subsection{Spatial Shift Unwrapping in SSE based FPP}

In order to work out how to unwrap the spatial shift, we can see how $u(x)$ is wrapped into $u_{w}(x)$. From Figure 3 (b) and (c), we observed the following:

- When $u(x)$ varies increasingly and reaches points $T_{0}, 2 T_{0}, 3 T_{0}, \ldots$ (that is, integer multiples of $T_{0}$ ), $u_{w}(x)$ exhibits a drop of $T_{0}$ with its value dropping from $T_{0}$ to 0.

- When $u(x)$ varies decreasingly and reaches the same points, that is, the integer multiples of $T_{0}, u_{w}(x)$ will jump from 0 to $T_{0}$.

Spatial shift unwrapping should reverse process from $u_{w}(x)$ to $u(x)$. In other words, when we observe a drop from $T_{0}$ to 0 , we should add $T_{0}$ to the wrapped result, and when we notice a jump from 0 to $T_{0}$, we should add $-T_{0}$. Assuming that the object has a continuous surface, and $u_{w}(x)$ is acquired in discrete form, that is, $u_{w}\left(x_{i}\right)$ $(i=0,2,3, \ldots ., N)$, spatial shift unwrapping should be carried out by the following procedure: 
Step 1: Initialization $u\left(x_{0}\right)=u_{w}\left(x_{0}\right)+k T_{0}$, where $k_{0}$ is determined by the height of the object at $x_{0}$. Because $u_{w}(x)<<T_{O}$, from Equations (12) and (13) we have $u\left(x_{0}\right)=\frac{d_{0} h\left(x_{0}\right)}{l_{0}}$ and $k=\operatorname{Integer}\left[\frac{u\left(x_{0}\right)}{T_{0}}\right]$

Step 2: Starting from $u_{w}\left(x_{0}\right)$ and for $u_{w}\left(x_{i}\right)$, where $i=0,2,3, \ldots ., N$;

If $u\left(x_{i}\right)$ increases followed by a drop of $T_{0}$, that is, $u_{w}\left(x_{i}\right)-u_{w}\left(x_{i-1}\right)=-T_{0}$, we should increase $k$ by 1 , that is, $k=k+1$;

else if $u\left(x_{i}\right)$ decreases followed by a jump of $T_{0}$, that is $u_{w}\left(x_{i}\right)-u_{w}\left(x_{i-1}\right)=T_{0}$, then decrease $k$ by 1 , that is, $k=k-1$;

otherwise, keep $k$ unchanged, that is, $k=k$;

Compute the unwrapped shift by $u(x)=u_{w}(x)+k T_{0}$

The above procedure is straight forward, but a number of issues must be resolved for its implementation in practice. Firstly, we should determine if $u_{w}\left(x_{i}\right)$ increases or decreases. Secondly we should be able to detect the sharp drop and jump. In ideal cases when surface is continuous, $s\left(x_{i}\right)$ and $d\left(x_{i}\right)$ are free of noise, these can be carried easily. However, in practice both $s\left(x_{i}\right)$ and $d\left(x_{i}\right)$ may contain noise, resulting in $u_{w}\left(x_{i}\right)$ corrupted by noise and disturbance. In order to eliminate the influence of noise and disturbance, we should make sure that $u_{w}\left(x_{i}\right)$ is as smooth as possible with respect to $x_{i}$. To this end we propose the following:

- Pre-processing of $s\left(x_{i}\right)$ and $d\left(x_{i}\right)$ by means of a digital filter in order to remove the noise and to smooth the waveform of $s\left(x_{i}\right)$ and $d\left(x_{i}\right)$. The challenges associated with pre-processing is to eliminate the unwanted noise and disturbance while keeping the original waveform of $s\left(x_{i}\right)$ and $d\left(x_{i}\right)$. Parameters of these filters should be selected with care.

- In order to determine the slop of $u_{w}\left(x_{i}\right)$, we firstly evaluate the difference of $u_{w}\left(x_{i}\right)$ over successive samples by $\Delta u_{w}\left(x_{i}\right)=u_{w}\left(x_{i}\right)-u_{w}\left(x_{i-1}\right)$, and then take the sign of the variance by $\delta_{i}=\operatorname{sign}\left\{\Delta u_{w}\left(x_{i}\right)\right\} \quad$ (where $\delta_{i}$ equals to 1,0 and -1 for $\Delta u_{w}\left(x_{i}\right)>0, \Delta u_{w}\left(x_{i}\right)=0$ and $\Delta u_{w}\left(x_{i}\right)<0$ respectively ). We carry out the following:

$$
\begin{aligned}
& \text { If } \frac{1}{M} \sum_{j=i-M}^{i-1} \delta_{i}>0 \text {, then } u_{w}\left(x_{i}\right) \text { increases, or } \\
& \text { If } \frac{1}{M} \sum_{j=i-M}^{i-1} \delta_{i}<0 \text {, then } u_{w}\left(x_{i}\right) \text { decreases. }
\end{aligned}
$$

In practice, a sharp jump or drop may transverse a few data samples on $u_{w}\left(x_{i}\right)$. Assuming that the jump or drop occurs within $L$ samples, we should use the following to detect the drop:

$$
\begin{aligned}
& \text { If } u_{w}\left(x_{i}\right)-u_{w}\left(x_{i-L}\right)>T_{2} \text {, then } u_{w}\left(x_{i}\right) \text { jumps, or } \\
& \text { If } u_{w}\left(x_{i}\right)-u_{w}\left(x_{i-L}\right)<-T_{2} \text {, then } u_{w}\left(x_{i}\right) \text { drops }
\end{aligned}
$$


where $T_{2}$ is the threshold which should be chosen based on the quality of $u_{w}\left(x_{i}\right)$. With the pre-processing of $s\left(x_{i}\right)$ and $d\left(x_{i}\right), u_{w}\left(x_{i}\right)$ is smooth enough and hence we choose $T_{2}=T_{0} / 2$.

\section{Experiments and Results}

In order to test the performance of the performance of the approach proposed in Section III, experiments were carried out in our laboratory. The experimental setup is shown in Figure 5. The digital projector used is HITACHI CP-X260, and camera is Duncan Tech MS3100. The digital camera is placed on top of the projector with a distance of $350 \mathrm{~mm}$. The distance between the camera lens and the reference plan is $1295 \mathrm{~mm}$. We used a dome set on a flat board as the object, shown in Figure 6(a), where the maximum height is $22.8 \mathrm{~mm}$. The diameter of the bottom surface of the dome is $99 \mathrm{~mm}$, and the thickness of the base board is $16 \mathrm{~mm}$. The resolution of the CCD camera is $1392 \times 1039$ pixels, and the filed of vision for CCD camera is $250 \mathrm{~mm} \times 187 \mathrm{~mm}$. Hence, the equivalent spatial resolution is $0.1796 \mathrm{~mm} / \mathrm{pixel}$.

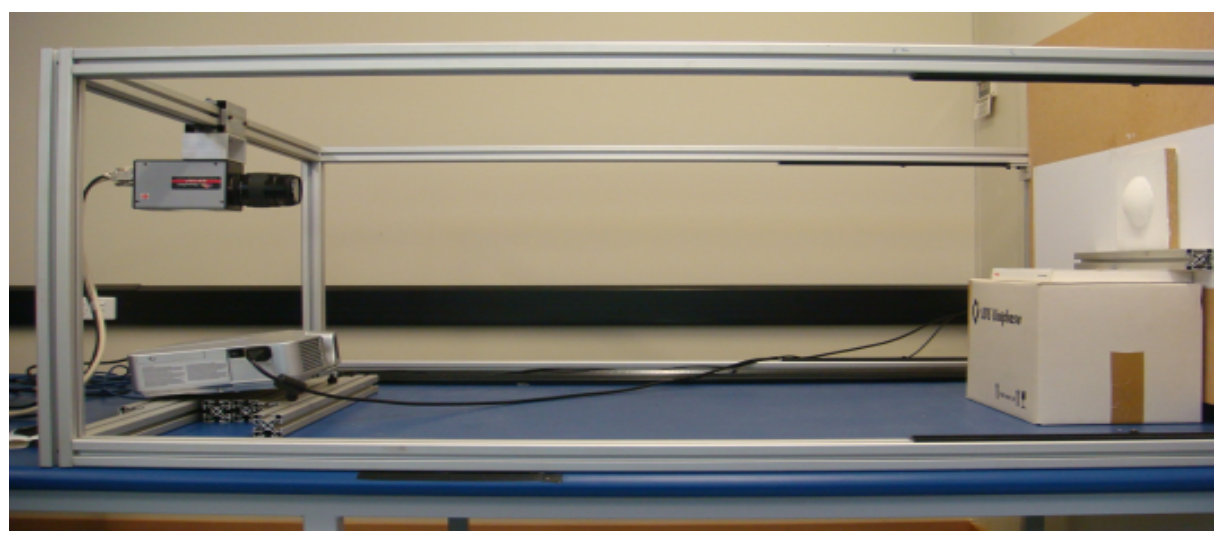

Figure 5. The experimental system setup

The captured fringe patterns on the reference plane and on the object surface are shown in Figures 6 (b) and 6 (c) respectively. We have 9 fringes, each covering 96 pixels.

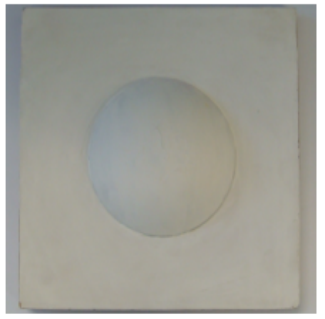

(a)

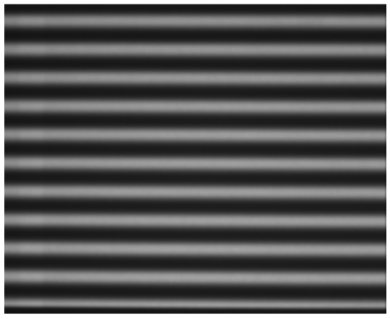

(b)

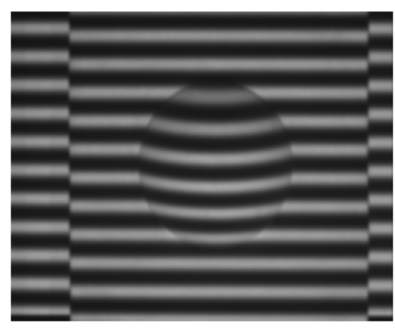

(c)

Figure 6. Fringe patterns observed 
In order to demonstrate the proposed approach, we look at a cross section of the fringe patterns. Figure 7 shows $s\left(x, y_{1}\right)$ and $d\left(x, y_{1}\right)$ respectively where $y_{1}=750$, which is the middle line of the dome. Obviously, both fringe patterns are distorted and corrupted by the noise.
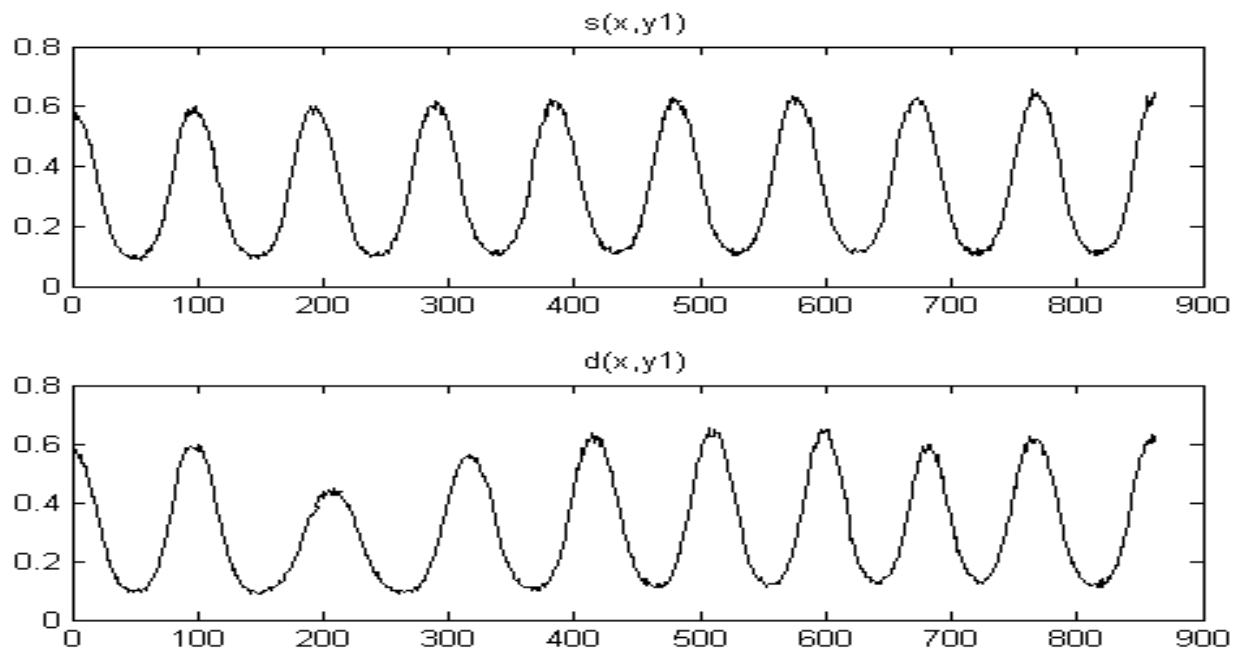

Figure 7. Fringe data acquired

In order to eliminate the influence of noise, we firstly employed a 128th order FIR low pass filter. Then we normalized the amplitude of each individual fringe period of $d\left(x, y_{1}\right)$ according to the corresponding fringe on $s\left(x, y_{1}\right)$. The results of pre-processing are shown in Figure 8.
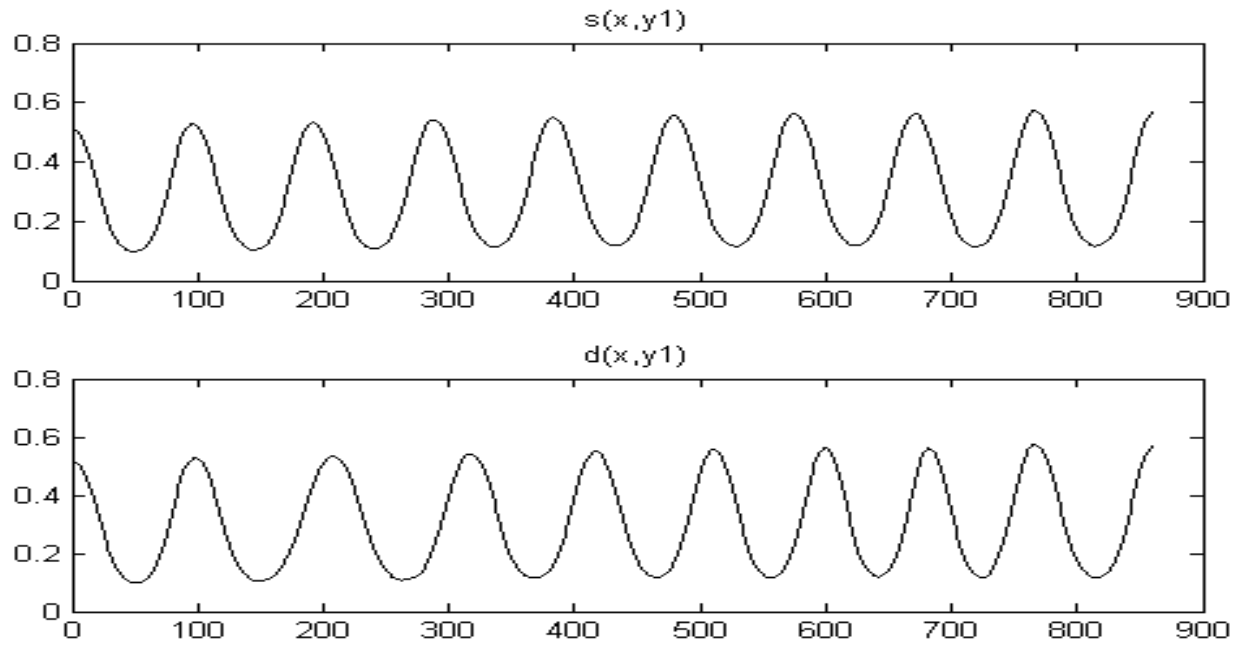

Figure 8. Fringe data pre-processed

The inverse function analysis approach [21] was employed to calculate $u\left(x, y_{1}\right)$. With this method, we firstly divided $s\left(x, y_{1}\right)$ and into segments, each covering a half fringe period either monotonically increasing or decreasing. For each individual segment, we found the inverse function using polynomial fitting, and then applied the inverse function to the corresponding segment on $d\left(x, y_{1}\right)$ to obtain the wrapped $u_{w}\left(x, y_{1}\right)$ in Figure 9 (a). With the proposed unwrapping approach we recovered $u\left(x, y_{1}\right)$ in Figure 9 (b), with which we obtained the height distribution $h\left(x, y_{1}\right)$ 
in Figure 9 (c). From Figure 9 (c), the max height of $h\left(x, y_{1}\right)$ is $23.08 \mathrm{~mm}$ and hence the measurement accuracy is $1.228 \%$. Therefore we can say that shape of the object can be successfully retrieved with the proposed approach.
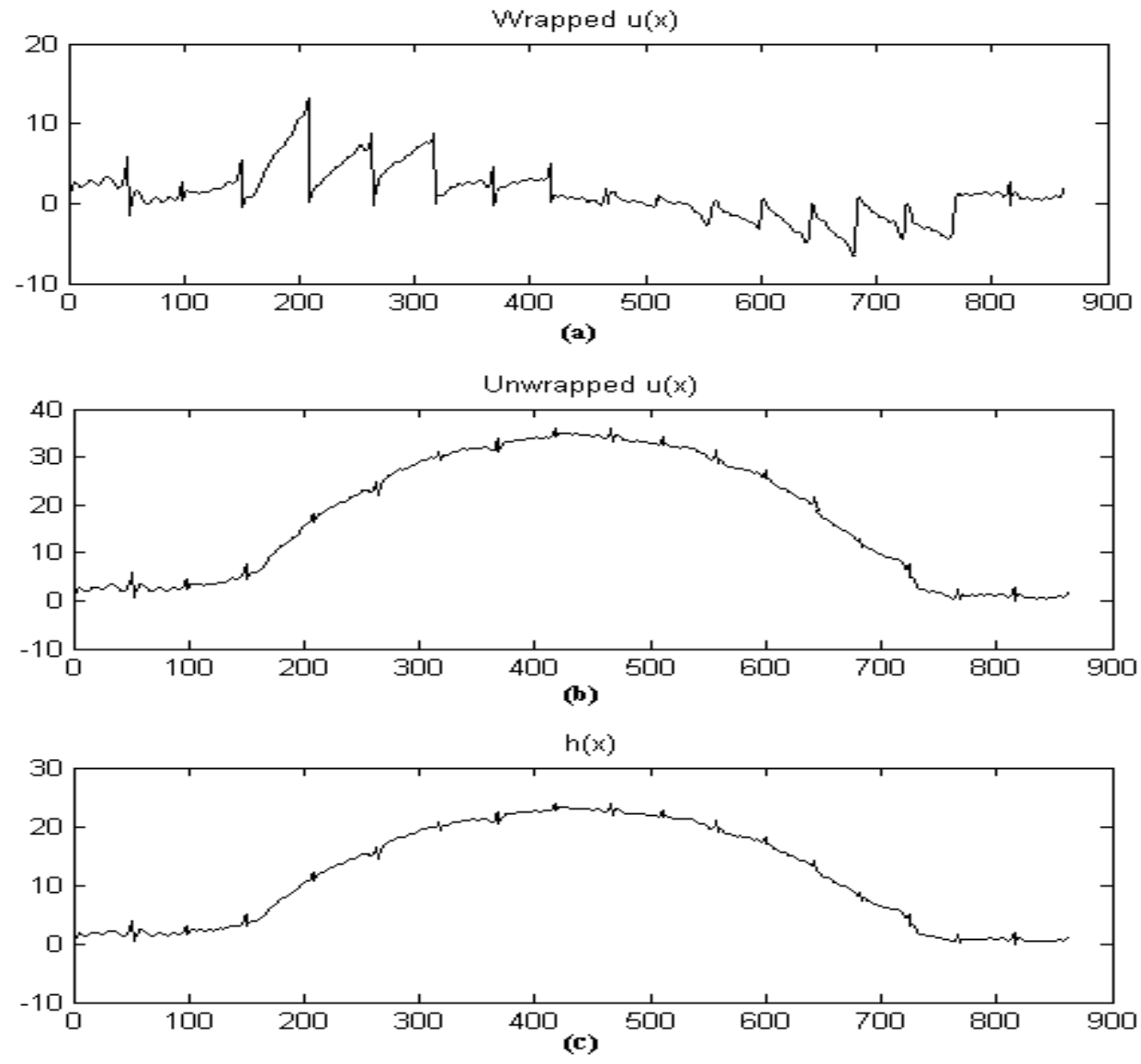

Figure 9. Spatial shift unwrapping and high distribution estimation

Then we employed the proposed for all $d\left(x, y_{1}\right)$ with $y_{1}=1,2, \ldots, 800$ and the 3D shape surface shape of the object was constructed shown in Figure 10.

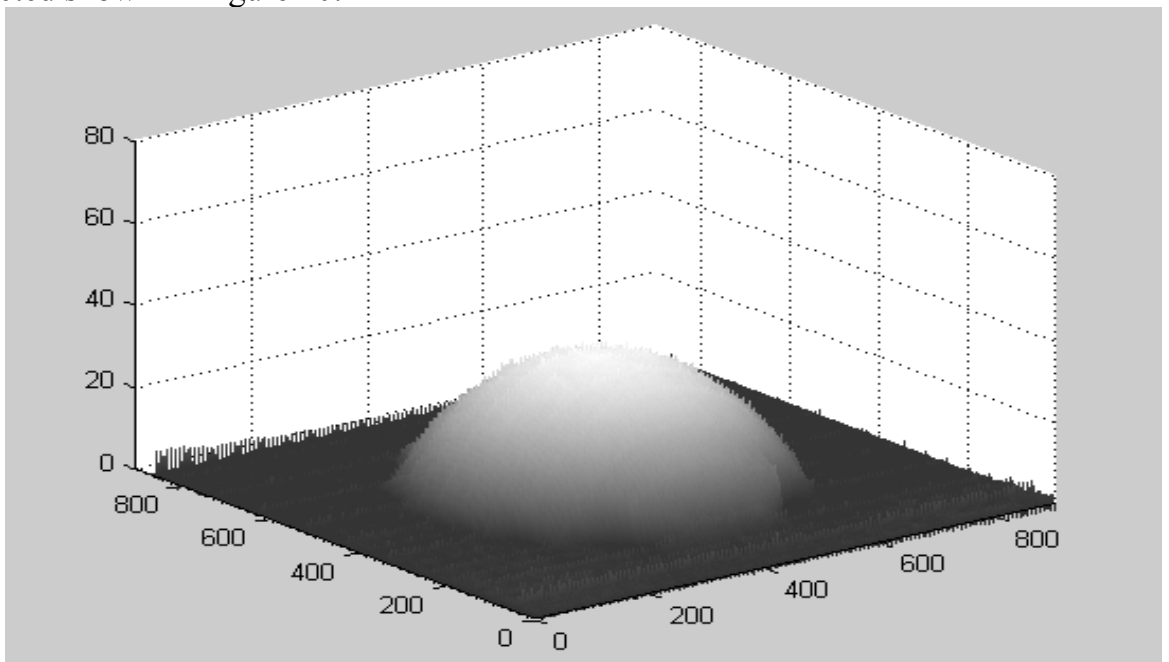

Figure 10. 3D Reconstruct Results

Proc. of SPIE Vol. 7432 743208-10 


\section{Conclusion}

In this paper, we studied the spatial shift wrapping problem associated with SSE-based FPP. The problem arises as the result of fringe reuse (that is, fringes periodic light intensity variance), and the spatial shift can only be identified without ambiguity with the range of a fringe width. We presented a technique to carry out spatial shift unwrapping to remedy the problem. In order to test the performance, we also carried out experiments on an object with simple hemisphere surface shape. The results have shown the effectiveness of the proposed unwrapping technique.

\section{REFERENCES}

[1] X. Su and W. Chen, "Fourier transform profilometry: a review," Opt. Lasers Eng. 35, 263-284, 2001.

[2] H. Zhang, M. J. Lalor, and D. R. Burton, "Spatiotemporal phase unwrapping for the measurement of discontinuous objects in dynamic fringe-projection phase-shifting profilometry," Applied Optics, vol. 38, pp. 3534-3541, June 1999.

[3] M. Halioua and H. C. Liu, "Optical three-dimensional sensing by phase measuring profilometry," Opt. Lasers Eng. 11, pp.185-215, 1989.

[4] J. Li, H. Su, and X. Su, "Two-frequency grating used in phase-measuring profilometry," Applied Optics, vol. 36, pp. 277-280, Janurary 1997.

[5] X. Su, L. Su, W. Li, and L. Xiang, "New 3D profilometry based on modulation measurement," Proceedings of SPIE, Vol. 3853, pp. 1-7, 1998.

[6] S. Toyooka and M. Tominga, "Spatial fringe scanning for optical phase measurement," Optics Communications, Vol. 51, pp. 68-70, 1984.

[7] S. Toyooka and Y. Iwaasa, "Automatic profilometry of 3-D diffuse objects by spatial phase detection," Applied Optics, vol. 25, no. 10, pp. 1630-1633, May 1986.

[8] R. Rodriguez-Vera and M. Servin, "Phase locked loop profilometry," Optics and Lasers Technology, vol. 26, no. 6, pp. 393-398, 1994.

[9] D. M. Meadows, W. . Johnson, and J. B. Allen, "Generation of surface contours by moiré patterns," Applied Optics, vol. 9, no. 4, pp. 942-947, April 1970.

[10] A. Asundi and Z. Wensen, "Unified calibration technique and its applications in optical triangular profilometry," Applied Optics, vol. 38, no. 16, pp. 3556-3561, June 1999.

[11] C. Wust and D. W. Capson, "Surface profile measurement using color fringe projection," MVA, vol. 4, pp. 193203, 1991.

[12] P. Huang, Q. Ho, F. Jin, and F. Chiang, "Colour-enhanced digitial fringe projection technique for high-speed 3-D surface contouring," Optics Engineering, vol. 38, pp. 1065-1071, 1999.

[13] A. J. Moore and F. Mendoza-Santoyo, "Phase demodulation in the space domain without a fringe carrier," Optics and Lasers in Engineering, vol. 23, pp. 319-330, 1995.

[14] J. Villa, M. Servin, and L. Castillo, "Profilometry for the measurement of 3-D object shapes based on regularized filters," Optics Communication, vol. 161, pp. 13-18, 1999.

[15] Y. Hu, J. Xi, E. Li, J. Chicharo, and Z. Yang, "Three-dimensional profilometry based on shift estimation of projected fringe patterns,” Applied Optics, vol. 45, no. 4, pp. 678-687, February 2006.

[16] Y. Hu, J. Xi, Z. Yang, E. Li, and J. Chicharo, "Generalized analysis model for fringe pattern profilometry," in IEEE Instrumentation and Measurement Conference (IMTC), Ottawa, Canada, May 2005, pp. 1951-1955.

[17] K. Itoh, "Analysis of the phase unwrapping algorithm", Applied Optics, Vol. 21, Issue 14, pp. 2470-2470, July 1982

${ }^{[18]}$ D. C. Ghiglia, M. D. Pritt, Two-Dimensional Phase Unwrapping: Theory, Algorithms, and Software. John Wiley \& Sons, 1998

[19] M. Takeda, H. Ina, and S. Kobayashi, "Fourier-transform method of fringe-pattern analysis for computer-based topography and interferometry," Journal of the Optical Society of America A, vol. 72, pp. 156-160, 1982.

[20] M. Takeda and K. Mutoh, "Fourier transform profilometry for the automatic measurement of 3-D object shapes," Applied Optics, vol. 22, pp. 3977-3982, 1983.

[21] Y. Hu, J. Xi, E. Li, J. Chicharo, and Z. Yang, "Fringe pattern profilometry based on inverse function analysis", Proceedings of 2005 International Symposium on Intelligent Signal Processing and Communication Systems (ISPACS 2005), 13-16 December 2005, pp.61-64. Copyright IEEE 2005. 